\title{
The Importance of Reporting Unexpected Drug Failure
}

\section{Luca Romanelli*, Emanuela Mhillaj and Vincenzo Cuomo}

Department of Physiology and Pharmacology V. Erspamer, University of Rome Sapienza, Rome, Italy

*Corresponding author: Luca Romanelli, Department of Physiology and Pharmacology V. Erspamer, University of Rome Sapienza, Rome, Italy, Tel: 390649912575; E-mail: luca.romanelli@uniroma1.it

Received date: February 04, 2016; Accepted date: February 06, 2016; Published date: February 10, 2016

Copyright: @ 2016 Romanelli L, et al. This is an open-access article distributed under the terms of the Creative Commons Attribution License, which permits unrestricted use, distribution, and reproduction in any medium, provided the original author and source are credited.

\section{Editorial}

Unexpected drug failure is a lack of therapeutic effect, which is not expected based on the characteristics of the drug and patient. Essentially, it may occur either for interactions (with other drugs or foods) or for biopharmaceutical issues. The risk arising from interactions is generally well characterised and physicians are well aware of it, and the importance of reporting cases of failure due to interactions is obvious. Less is known about the mechanisms and potential risk deriving from biopharmaceutical problems, and we will therefore focus on this issue. Biopharmaceutics describes the influence of the physical/chemical properties of the drug and drug product on the rate and extent of systemic drug absorption. Because after oral administration of solid pharmaceutical forms drugs must dissolve in gastrointestinal fluids before being absorbed through the intestinal epithelium, biopharmaceutical problems essentially arise from an insufficient dissolution performance. A typical situation where an insufficient dissolution may cause unexpected drug failure is when a patient is switched from one drug product to a different product. Different drug products with the same active substance may differ in manufacture and excipient composition.

These differences may cause a different dissolution performance in vivo, leading to different rate and/or extent of absorption. Therefore, drug products are considered to be interchangeable only if their bioequivalence has been demonstrated through pharmacokinetic studies in vivo and/or comparative dissolution studies in vitro. However, the bioavailability of two bioequivalent drug may differ, even though only to a limited extent, and this may lead to unexpected drug failure following a switch, appearing as sudden loss of a full therapeutic effect in patients previously responding to the drug. An impressive case report described the consequences of switching 25 patients with a stable response to branded clozapine (Clozaril') to a generic clozapine [1]. Seven of the 25 patients experienced a relapse, defined as the reemergence of previously controlled psychotic symptoms that either required hospitalization or for which hospitalization was considered. Six additional patients experienced mild exacerbations of symptoms. Thus, $52 \%$ of the 25 patients showed at least some sign of deterioration after the switch to the generic formulation of clozapine. In the 7 patients experiencing a relapse, no improvement was achieved by increasing the dose of generic clozapine. However, all 7 patients rapidly improved when Clozaril ${ }^{\circ}$ was reinstated. A clinical study investigating the clinical effects of a randomized switch of patients from Clozaril to generic clozapine confirmed that the switch was associated with an increased risk of therapeutic failure [2]. Generic clozapine had been authorised based on a bioequivalence study performed in healthy volunteers using a sub-therapeutic dose of $12.5 \mathrm{mg}$ because of safety concerns. The assumption was that the bioequivalence would also be valid with the much higher therapeutic doses. These and other reports indicated that this was not the case and eventually led the FDA to change the protocol of bioequivalence studies for generic clozapine, which currently prescribes the use of a therapeutic dose of $100 \mathrm{mg}$ in patients. Nowadays, generic clozapine is considered to be therapeutically equivalent to Clozaril ${ }^{\circ}[3]$.

The risk of therapeutic failure as a consequence of switching during therapy is considered to be particularly relevant for anti-epileptic drugs (AEDs) [4,5]. AEDs are considered to be narrow therapeutic index drugs, because even small deviations in bioavailability have the potential to result in loss of seizure control in some patients. Therefore, while initiating an innovator or generic antiepileptic drug will provide similar efficacy, tolerability, and safety, switching from one form to the other may be associated with therapeutic failure. There have been several reports of breakthrough seizures after switching from one version of an AED to another, (whether the switch was brand-togeneric, generic-to-brand, or generic-to-generic. on generic substitution) [4]. Subsequent pharmacoepidemiological studies found that generic substitution AEDs was associated with significantly greater risk of epilepsy-related medical events and requiring urgent seizure-related care $[6,7]$.

As a result of these reports and studies, Epilepsy Foundation has developed serious concerns about policies that permit or require AED substitutions without the consent of the doctor and patient [8]. Epilepsy Foundation has asked the FDA to "consider issuing a limited advisory to patients and physicians regarding potential problems with switching between different medication formulations (whether generic to generic or generic to brand) for some people with epilepsy". Specifically, Epilepsy Foundation hopes that the FDA would at least advise physicians that they should consider monitoring the blood levels of patients before making any subsequent changes in the patient's treatment regimen and that, for some patients, no switching should occur except with the oversight, monitoring, and consent of the physician and patient. These concerns about switch are shared by other scientific associations.

The Italian League against Epilepsy (LICE) even issued a guideline not recommending the switch in patients with a complete clinical control. From a regulatory perspective the issue of switching is very complex. In fact, in 1999 the FDA issued a draft Guidance introducing the concept of 'switchability', and the related criteria for bioequivalence studies, but the Guidance was later substituted with a new one, currently in use, which does not address directly the problem of switchability. However, regulatory agencies have revised their bioequivalence guideline in order to reduce the risk of therapeutic failure for narrow therapeutic index (NTI) drugs. The current EU and Canadian Guidelines on bioequivalence have introduced the concept that the bioequivalence interval should be tightened to $90-111 \%$ (instead of the usual 80.0-125.0\% interval) for NTI drugs. The FDA has published product-specific bioequivalence draft guidance for two NTI drugs (warfarin sodium tablet and tacrolimus capsule) recommending 
Page 2 of 2

to apply a study protocol and statistical approach different from those generally used to demonstrate the bioequivalence.

There may be a risk of therapeutic failure also when a patient is switched from one formulation to a different formulation of a same drug product. Different formulations of the same drug product differ in their pharmaceutical form, for example tablets vs. oral solution, and may have a different bioavailability. A case of serious therapeutic failure caused by switching form one formulation to another has been recently reported by the FDA [9]. The antifungal drug Noxafil (posaconazole) is available in two oral formulations, delayed-release tablet and oral suspension, with the tablet having a higher bioavailability than the suspension.

In the case report, a patient was taking Noxafil delayed-release tablets for prophylaxis of invasive Aspergillus and Candida infections, but the pharmacy replaced the tablets with the oral suspension. The patient was reported to have later died from a stroke related to an invasive Aspergillus infection. This and other reports led the FDA to release a Safety Communication cautioning about dosing errors when switching between the different oral formulations. In addition, the manufacturer of Noxafil revised the prescribing information and the patient information in the drug label to alert patients and their health care professionals that the two oral formulations of Noxafil cannot be substituted for each other.

It should be considered that unexpected failure due to biopharmaceutical issues is not necessarily related to a switch but may also arise in patients with a particular gastrointestinal physiology. An explicative example is given by the report of Yamamoto [10], who observed that three patients with hypothyroidism did not achieve the therapeutic goal, i.e., normalization of serum thyrotropin (TSH) despite taking high doses of levothyroxine (LT4) as tablets. History of gastrointestinal illnesses, signs and symptoms of such a condition, and concurrent medications known to affect the absorption of LT4 from the gut were absent. The patients' TSH levels normalized when the tablets were taken after being pulverized. This clearly indicates that in these 3 patients the drug dissolved too slowly from the tablets, thus leading to insufficient bioavailability.

Pulverization increased the rate of dissolution and, consequently, the bioavailability. Noteworthy, Yamamoto reported that during the period of the clinical study with the 3 patients, he treated 105 adult patients with primary hypothyroidism, with no problems in normalizing their TSH with standard LT4 doses, indicating that the problem arose from the interaction between the particular physiological characteristics of some patients and the pharmaceutical performance of the drug product. Dr. Yamamoto concluded that for patients with hypothyroidism that require more than the optimal dose of $200 \mathrm{mg} /$ day in order to keep the serum TSH levels within the normal ranges, pulverization of the tablets is worth consideration. It could be could be anticipated that a liquid LT4 formulation should perform even better than pulverized tablets. Liquid formulations of LT4 are now marketed, and clinical trials suggest that they may actually be superior to the tablets in maintaining the euthyroid state [11].

In conclusion, physicians, pharmacists, and patients should always keep in mind the possibility that unexpected drug failure may be due to biopharmaceutical issues. Reporting these cases of unexpected drug failure may provide valuable information for finding a solution for notresponder patients. As we have seen, reporting unexpected drug failures can also lead to development of revised guidelines or recommendations from regulatory agencies or scientific associations, thus increasing the safe use of drugs. In some cases, it may even concur to encourage pharmaceutical companies to develop new drug formulations, as in the case of liquid LT4.

\section{References}

1. Mofsen R, Balter J (2001) Case reports of the reemergence of psychotic symptoms after conversion from brand-name clozapine to a generic formulation. Clin Ther 23: 1720-1731.

2. Kluznik JC, Walbek NH, Farnsworth MG, Melstrom K (2001) Clinical effects of a randomized switch of patients from clozaril to generic clozapine. J Clin Psychiatry 62 Suppl 5: 14-17.

3. Bobo WV, Stovall JA, Knostman M, Koestner J, Shelton RC (2010) Converting from brand-name to generic clozapine: A review of effectiveness and tolerability data. Am J Health Syst Pharm 67: 27-37.

4. Crawford P, Feely M, Guberman A, Kramer G (2006) Are there potential problems with generic substitution of antiepileptic drugs? A review of issues. Seizure 15: 165-176.

5. Talati R, Scholle JM, Phung OP, Baker EL, Baker WL, et al. (2012) Efficacy and safety of innovator versus generic drugs in patients with epilepsy: a systematic review. Pharmacotherapy 32: 314-322.

6. Labiner DM, Paradis PE, Manjunath R, Duh MS, Lafeuille MH, et al. (2010) Generic antiepileptic drugs and associated medical resource utilization in the United States. Neurology 74: 1566-1574.

7. Zachry WM III, Doan QD, Clewell JD, Smith BJ (2009) Case-control analysis of ambulance, emergency room, or inpatient hospital events for epilepsy and antiepileptic drug formulation changes. Epilepsia 50: 493-500.

8. Epilepsy Foundation comments on generic drugs user fee amendments.

9. Drug Safety Communication-Dosing Errors when Switching between Different Oral Formulations; Label Changes Approved.

10. Yamamoto T (2003) Tablet formulation of levothyroxine is absorbed less well than powdered levothyroxine. Thyroid 13: 1177-1181.

11. Negro R, Valcavi R, Agrimi D, Toulis KA (2014) Levothyroxine liquid solution versus tablet for replacement treatment in hypothyroid patients. Endocr Pract 20: 901-906. 